\title{
Protein kinase CK2: from structures to insights
}

\author{
K. Niefind $\cdot$ J. Raaf $\cdot$ O.-G. Issinger
}

Published online: 20 August 2009

(C) Birkhäuser Verlag, Basel/Switzerland 2009

Erratum to: Cell. Mol. Life Sci. 66 (2009) 1800-1816

DOI 10.1007/s00018-009-9149-8

Due to errors in the editorial process most references in Table 1 of K. Niefind, J. Raaf and O.-G. Issinger,
Cell. Mol. Life Sci. 66 (2009), 1800-1816 (DOI 10.1007/s00018-009-9149-8), are wrong. The authors and the publishers apologize for any inconvenience. The correct Table 1 reads as follows:

Table 1 CK2 structures available at the Protein Data Bank [18] ranked according to resolution

\begin{tabular}{|c|c|c|c|c|c|c|}
\hline $\begin{array}{l}\text { Protein construct/ } \\
\text { designation in the text }\end{array}$ & Species & Main ligands & $\begin{array}{l}\text { Resol. } \\
(\AA)\end{array}$ & $\begin{array}{l}\text { Exp. data } \\
\text { available? }\end{array}$ & $\begin{array}{l}\text { PDB } \\
\text { code }\end{array}$ & Ref. \\
\hline$h s \mathrm{CK} 2 \alpha^{1-335}$ & H. sapiens & $\begin{array}{l}\text { 3-Methyl-1,6,8-trihydroxyanthraquinone } \\
\text { (emodin) }\end{array}$ & 1.50 & Yes & 3BQC & {$[34]$} \\
\hline$h s \mathrm{CK} 2 \alpha^{1-335}$ & H. sapiens & $\begin{array}{l}\text { 5,6-Dichloro- } 1-\beta \text {-D-ribofuranosyl-1H- } \\
\text { benzimidazole (DRB) }\end{array}$ & 1.56 & Yes & $3 \mathrm{H} 30$ & {$[30]$} \\
\hline$h s \mathrm{CK} 2 \alpha^{1-335}$ & H. sapiens & $\begin{array}{l}\text { Adenosine } 5^{\prime}-(\beta, \gamma \text {-imido }) \text { triphosphate } \\
\quad(\text { AMPPNP }), \text { sulphate ions }\end{array}$ & 1.61 & Yes & 2PVR & {$[35]$} \\
\hline$h s \mathrm{CK} 2 \alpha^{1-335} \mathrm{~V} 66 \mathrm{~A} / \mathrm{M} 163 \mathrm{~L}$ & H. sapiens & $\begin{array}{l}\text { Adenosine } 5^{\prime}-(\beta, \gamma \text {-imido }) \text { triphosphate } \\
\quad(\text { AMPPNP }), \text { glycerol }\end{array}$ & 1.66 & Yes & 3BW5 & {$[30,33]$} \\
\hline$z m \mathrm{CK} 2 \alpha$ & Zea mays & $\begin{array}{l}\text { [5-Oxo-5,6-dihydroindolo-(1,2-a)quinazolin-7- } \\
\text { yl]-acetic acid }\end{array}$ & 1.68 & No & 1OM1 & {$[36]$} \\
\hline$z m \mathrm{CK} 2 \alpha$ & Zea mays & 5,8-Diamino-1,4-dihydroxyanthrachinon & 1.70 & No & $1 \mathrm{M} 2 \mathrm{R}$ & {$[37]$} \\
\hline$z m \mathrm{CK} 2 \alpha$ & Zea mays & $\begin{array}{l}\text { 2-(Cyclohexylmethylamino)-4- } \\
\text { (phenylamino)pyrazolo[1,5-a] }[1,3,5] \text { triazine- } \\
\text { 8-carbonitrile }\end{array}$ & 1.70 & No & $2 \mathrm{PVJ}$ & {$[25]$} \\
\hline
\end{tabular}

The online version of the original article can be found under doi:10.1007/s00018-009-9149-8.

\section{K. Niefind $(\bowtie) \cdot$ J. Raaf}

Universität zu Köln, Department für Chemie, Institut für Biochemie, Zülpicher Straße 47, 50674 Köln, Germany

e-mail: Karsten.Niefind@uni-koeln.de

O.-G. Issinger

Syddansk Universitet,

Institut for Biokemi og Molekylær Biologi,

Campusvej 55, 5230 Odense, Denmark 
Table 1 continued

\begin{tabular}{|c|c|c|c|c|c|c|}
\hline $\begin{array}{l}\text { Protein construct/ } \\
\text { designation in the text }\end{array}$ & Species & Main ligands & $\begin{array}{l}\text { Resol. } \\
(\AA)\end{array}$ & $\begin{array}{l}\text { Exp. data } \\
\text { available? }\end{array}$ & $\begin{array}{l}\text { PDB } \\
\text { code }\end{array}$ & Ref. \\
\hline$h s \mathrm{CK} 2 \beta^{1-182}$ & H. sapiens & - & 1.74 & No & 1QF8 & [7] \\
\hline$z m \mathrm{CK} 2 \alpha$ & Zea mays & $\begin{array}{l}\text { 4,5,6,7-Tetrabromo- } N, N \text {-dimethyl- } 1 \mathrm{H} \text { - } \\
\text { benzimidazol-2-amine }\end{array}$ & 1.77 & No & $1 \mathrm{ZOE}$ & [38] \\
\hline$z m \mathrm{CK} 2 \alpha$ & Zea mays & 1,8-Dihydroxy-4-nitro-xanthen-9-one & 1.79 & No & 1M2Q & [37] \\
\hline$z m \mathrm{CK} 2 \alpha$ & Zea mays & 4,5,6,7-Tetrabromo-benzimidazole & 1.81 & No & $2 \mathrm{OXY}$ & [39] \\
\hline$z m \mathrm{CK} 2 \alpha$ & Zea mays & $\begin{array}{l}\text { 5,6,7,8-Tetrabromo-1-methyl-2,3-dihydro- } 1 \mathrm{H} \text { - } \\
\text { imidazo[1,2-a] benzimidazole }\end{array}$ & 1.81 & No & $1 \mathrm{ZOH}$ & [38] \\
\hline$z m \mathrm{CK} 2 \alpha$ & Zea mays & $\begin{array}{l}\text { 3,8-Dibromo-7-hydroxy-4-mmethyl-2H- } \\
\text { chromen-2-one }\end{array}$ & 1.85 & Yes & 2QC6 & [40] \\
\hline$z m \mathrm{CK} 2 \alpha$ & Zea mays & Probably benzamidine & 1.86 & Yes & $1 \mathrm{LPU}$ & [33] \\
\hline$z m \mathrm{CK} 2 \alpha$ & Zea mays & $\begin{array}{l}\text { Adenosine } 5^{\prime} \text { - }(\beta, \gamma \text {-imido)triphosphate } \\
\quad \text { (AMPPNP) }\end{array}$ & 1.86 & Yes & 1LP4 & [33] \\
\hline$z m \mathrm{CK} 2 \alpha$ & Zea mays & $\begin{array}{l}\text { 2-(4-Ethylpiperazin-1-yl)-4-(phenylamino) } \\
\quad \text { pyrazolo[1,5-a] }[1,3,5] \text { triazine-8-carbonitrile }\end{array}$ & 1.90 & No & 2PVL & [25] \\
\hline$z m \mathrm{CK} 2 \alpha$ & Zea mays & $\begin{array}{l}\text { 2-(4-Chlorobenzylamino)-4-(phenylamino) } \\
\text { pyrazolo[1,5-a] }[1,3,5] \text { triazine-8-carbonitrile }\end{array}$ & 1.90 & No & $2 \mathrm{PVK}$ & [25] \\
\hline$h s \mathrm{CK} 2 \alpha^{1-335}$ & H. sapiens & $\begin{array}{l}\text { 3-Methyl-1,6,8-trihydroxyanthraquinone } \\
\text { (emodin) }\end{array}$ & 1.95 & Yes & $3 \mathrm{C} 13$ & [34] \\
\hline$z m \mathrm{CK} 2 \alpha$ & Zea mays & Probably benzamidine & 2.00 & Yes & 1LR4 & [33] \\
\hline$z m \mathrm{CK} 2 \alpha$ & Zea mays & 1,8-Dihydroxy-4-nitro-anthrachinon & 2.00 & No & $1 \mathrm{M} 2 \mathrm{P}$ & [37] \\
\hline$z m \mathrm{CK} 2 \alpha$ & Zea mays & $\begin{array}{l}N \text {-(3-(8-Cyano-4-(phenylamino)pyrazolo[ } 1,5- \\
\quad \text { a] }[1,3,5] \text { triazin-2-ylamino) phenyl)acetamide }\end{array}$ & 2.00 & No & $2 \mathrm{PVN}$ & [25] \\
\hline$z m \mathrm{CK} 2 \alpha$ & Zea mays & $\begin{array}{l}\text { 4-(2-(1H-Imidazol-4-yl)ethylamino)-2- } \\
\text { (phenylamino) pyrazolo[1,5-a][1,3,5]triazine- } \\
\text { 8-carbonitrile }\end{array}$ & 2.00 & No & $2 \mathrm{PVM}$ & [25] \\
\hline$z m \mathrm{CK} 2 \alpha$ & Zea mays & - & 2.18 & No & $1 \mathrm{JAM}$ & [41] \\
\hline$z m \mathrm{CK} 2 \alpha$ & Zea mays & 4,5,6,7-Tetrabromo-2-benzotriazole & 2.19 & No & $1 J 91$ & [41] \\
\hline$z m \mathrm{CK} 2 \alpha$ & Zea mays & $\begin{array}{l}\text { Adenosine } 5^{\prime} \text { - }(\beta, \gamma \text {-imido)triphosphate } \\
\quad \text { (AMPPNP) }\end{array}$ & 2.20 & Yes & 1DAW & [32] \\
\hline$z m \mathrm{CK} 2 \alpha$ & Zea mays & $\begin{array}{l}\text { Guanosine } 5^{\prime} \text { - }(\beta, \gamma \text {-imido)triphosphate } \\
\text { (GMPPNP) }\end{array}$ & 2.20 & Yes & 1DAY & [32] \\
\hline$z m \mathrm{CK} 2 \alpha$ & Zea mays & $\begin{array}{l}N, N^{\prime} \text {-Diphenylpyrazolo }[1,5-\mathrm{a}][1,3,5] \text { triazine- } \\
\text { 2,4-diamine }\end{array}$ & 2.20 & No & $2 \mathrm{PVH}$ & [25] \\
\hline$h s \mathrm{CK} 2 \alpha^{1-335}$ & H.sapiens & Glycerol & 2.30 & Yes & 3FWQ & [42] \\
\hline$z m \mathrm{CK} 2 \alpha$ & Zea mays & 4,5,6,7-Tetrabromo-1H,3H-benzimidazole-2-one & 2.30 & No & $1 \mathrm{OXD}$ & [39] \\
\hline$z m \mathrm{CK} 2 \alpha$ & Zea mays & $\begin{array}{l}\text { 4,5,6,7-Tetrabromo- } 1 \mathrm{H}, 3 \mathrm{H} \text {-benzimidazole-2- } \\
\text { thion }\end{array}$ & 2.30 & No & $2 \mathrm{OXX}$ & [39] \\
\hline$z m \mathrm{CK} 2 \alpha$ & Zea mays & $\begin{array}{l}\text { 4,5,6,7-Tetrabromo-2-(methylsulphanyl)- } 1 \mathrm{H}- \\
\text { benzimidazole }\end{array}$ & 2.30 & No & $1 \mathrm{ZOG}$ & [38] \\
\hline$h s \mathrm{CK} 2 \alpha^{1-329} \mathrm{E} 27 \mathrm{~A} / \mathrm{K} 76 \mathrm{~N}^{*}$ & H. sapiens & - & 2.40 & Yes & 1NA7 & [43] \\
\hline$h s \mathrm{CK} 2 \alpha^{1-335}$ & H. sapiens & $\begin{array}{l}\text { Adenosine } 5^{\prime}-(\beta, \gamma \text {-imido)triphosphate } \\
\quad \text { (AMPPNP) }\end{array}$ & 2.50 & Yes & 1PJK & [44] \\
\hline$z m \mathrm{CK} 2 \alpha$ & Zea mays & 3-Methyl-1,6,8-trihydroxyanthraquinone & 2.63 & No & 1F0Q & [45] \\
\hline$h s \mathrm{CK} 2 \beta^{1-193}$ & H. sapiens & - & 2.80 & Yes & 3EED & [27] \\
\hline$x l \mathrm{CK} 2 \beta^{1-178}$ & X. laevis & p21WAF1 peptide & 2.89 & Yes & $1 \mathrm{RQF}$ & [46] \\
\hline$r n \mathrm{CK} 2 \alpha^{1-335}$ & R. norvegicus & Sulphate ions & 3.00 & Yes & 2R7I & - \\
\hline$\left(h s \mathrm{CK} 2 \alpha^{1-337}\right)_{2} /(h s \mathrm{CK} 2 \beta)_{2} *$ & H. sapiens & $\begin{array}{l}\text { Adenosine } 5^{\prime} \text { - }(\beta, \gamma \text {-imido)triphosphate } \\
\text { (AMPPNP) }\end{array}$ & 3.10 & Yes & $1 \mathrm{JWH}$ & {$[8]$} \\
\hline$r n \mathrm{CK} 2 \beta^{1-193^{*}}$ & R. norvegicus & - & 3.10 & Yes & 2R6M & - \\
\hline$z m \mathrm{CK} 2 \alpha$ & Zea mays & Adenosine monophosphate (AMP) & 3.15 & No & 1DS5 & [16] \\
\hline
\end{tabular}

*In these cases the status of the respective C-terminus is unclear. The indicated C-termini were not introduced genetically but presumably formed by spontaneous degradation 